\title{
TUBERCULOUS ABDOMINAL ABSCESS IN AN HIV-INFECTED MAN: NEITHER INFECTION PREVIOUSLY DIAGNOSED
}

\author{
Kuo-Yao Kao, $M D$ \\ Tsung-I Hung, $M D$ \\ Department of General Surgery, Shin Kong Wo Ho-Su Memorial Hospital, Taipei, Taiwan
}

\begin{abstract}
A 38-year-old man had a 1-week history of right lower quadrant abdominal pain; the initial impression was that he had diverticulitis of the ascending colon with an intra-abdominal abscess. Signs of peritonitis mandated an immediate right hemicolectomy. The unusual location of the abscess and the patient's unusual postoperative course suggested that he might also have a systemic disease. Testing for HIV infection was positive. After 2 weeks in hospital, he was treated as an outpatient for both tuberculosis and HIV with a favourable outcome.
\end{abstract}

In Taiwan a pre-operative HIV test is not performed routinely, and the HIV seroprevalence in surgical patient populations is unknown. Surgeons should keep the possibility of HIV infection in mind in a patient with an unusual clinical course.

The number of new cases of human immunodeficiency virus (HIV) infection in Taiwan peaked in 2005, and it is still a serious problem. ' Tuberculosis (TB), especially extrapulmonary $\mathrm{TB}^{\mathrm{B}}$, is also common in Taiwan ${ }^{1}$ and is commonly associated with HIV infection. ${ }^{2}$ When a patient urgently needs surgery, as can occur with perforation of an intestine or appendix, there is no time for definitive testing for HIV and/or TB.

We report a case in which a patient required immediate surgery for what appeared to be an abscess with peritonitis from a perforated diverticulum of the ascending colon. The patient was unaware that he had both extrapulmonary TB and HIV. The TB was discovered by the pathologist on examination of the surgical specimen, and the HIV was discovered because the patient's postoperative condition suggested a systemic disease. The case illustrates that despite co-occurrence of the two diseases surgery can be successful, recovery can be similar to that expected in a patient without the diseases, and patient outcome can be good if anti-TB and antiretroviral therapies are started almost immediately. The case is also consistent with previous reports that patients with HIV undergoing surgery have similar conditions to HIV-negative patients and that the results of treatment are equivalent. ${ }^{3}$

\section{CASE REPORT}

A 38-year-old man who had been well without systemic disease and neither drank alcohol nor smoked had experienced dull, right lower quadrant abdominal pain for 1 week, and fever with chills for 2 days. He had no other associated symptoms such as nausea, vomiting or diarrhoea. He sought help at the emergency department, where physical examination showed rebound tenderness at the right lower quadrant of the abdomen, with muscle guarding and rigidity. The white blood cell count was $8 \times 10^{9} / l$, with $11 \%$ band-form neutrophils. An elevated C-reactive protein level $(5.77 \mathrm{mg} / \mathrm{dl})$ was noted. After he was admitted on 29 April 2006, an abdominal computed tomography scan revealed an ill-defined mass in the right lower quadrant anterior to the right psoas muscle; it was suspected to be an abscess. There were also several small abscesses in the omentum, paracolic gutter and mesentery of the ascending colon (Fig. 1). The clinical impression was that he had perforated diverticulitis with intra-abdominal abscess, and he was immediately operated on. At surgery the abscess was found to be at the ascending colon mesentery. A right hemicolectomy was performed because of the high level of suspicion of a perforated diverticulum (Fig. 2). Postoperatively, the patient had a high fever for 3 days even with intensive treatment with a secondgeneration cephalosporin (cefmetazole $1 \mathrm{~g}$ 8-hourly). A systemic disease was suspected because the unusual clinical profile, including the location of the abscess and the pathological findings, did not correspond with the patient's general condition. HIV infection was considered, and a Western blot test was positive for HIV. The patient's CD4 cell count, measured by flow cytometry, was 306/ $\mu$ l, and his CD8 cell count was 677/ $\mu \mathrm{l}$. The HIV viral load was measured by indirect enzyme- 


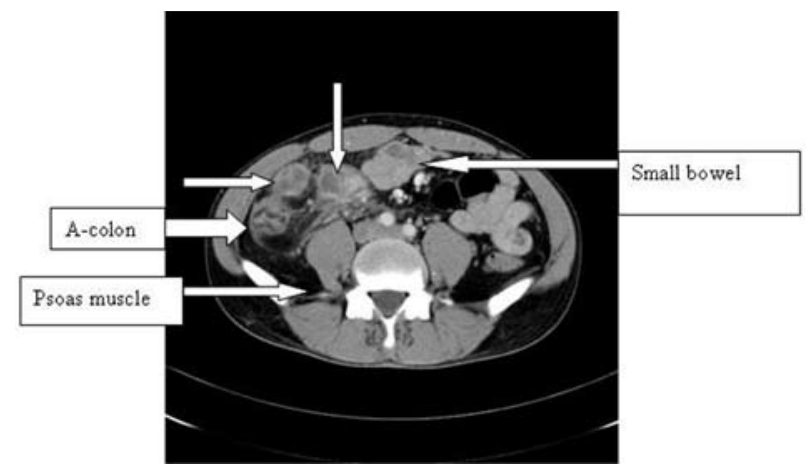

Fig. 1. Pre-operative computed tomography scan of the abdomen. The largest abscess is located at the ascending colon mesentery, anterior to the right psoas muscle (vertical arrow). There are several smaller abscesses over the omentum, and paracolic gutter (horizontal arrow).

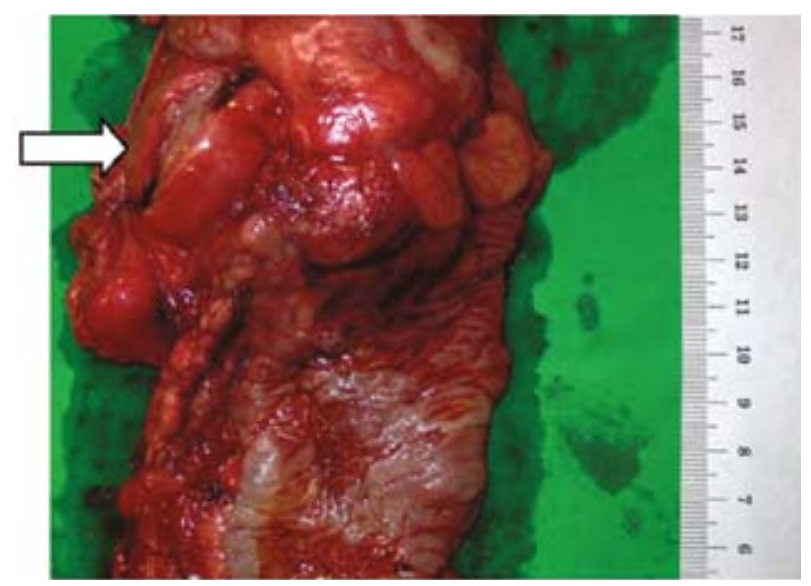

Fig. 2. The largest abscess after excision. This abscess was found adjacent to the ascending colon (arrows).

linked immunosorbent assay (ELISA) and found to be 6790 copies/ml.

The final diagnosis was HIV infection coincident with the histologically confirmed Mycobacterium tuberculosis colitis and abscess formation. Anti-TB drugs (rifampin + ethambutol) were started soon after the patient resumed oral intake. He was discharged on 11 May 2006, 14 days after admission, at which time highly active antiretroviral therapy (HAART) was started, including abacavir, efavirenz and lamivudine. Anti-TB drugs were given continuously in the outpatient department for 18 months; the regimen included isoniazid, rifampin, ethambutol and pyrazinamide. The patient was followed up until August 2009 with a favourable outcome; at that visit he had a viral load of $<400$ copies/ml, a CD4 count of 332 cells/ $\mu$ land a CD8 count of 632 cells/ $\mu$ l.

\section{DISCUSSION}

Our patient was unaware that he had TB and HIV infection, and because he urgently needed surgery we did not have time to perform laboratory tests to diagnose these infections. Nevertheless, he quickly recovered from the surgery and was discharged from the hospital 2 weeks after admission. Outpatient treatment for the TB and HIV had a favourable outcome.
TB has become a major cause of death and disability in many parts of the world, especially in developing countries. ${ }^{4,5}$ The disease can affect almost any body system, although most cases are pulmonary. ${ }^{6}$ In the USA, cases of extrapulmonary TB increased from $13.5 \%$ of all reported TB cases in 1975 to $21.0 \%$ in 2006. ${ }^{7}$ A study in Taiwan found that extrapulmonary TB cases increased from $23 \%$ to $27 \%$ from 1996 to $2003 .{ }^{8}$ Abdominal TB is a rare manifestation of extrapulmonary $T B$, with a prevalence of around $3 \% .{ }^{6}$ It may involve the gastrointestinal tract, peritoneum, mesenteric lymph nodes, genito-urinary tract, or other solid organs. ${ }^{4,6,9}$

Abdominal tuberculosis frequently poses a diagnostic challenge because specimens may be difficult to obtain, and the concentration of organisms may be low. Sanai and Bzeizi compiled data from 39 studies and found that the sensitivities of various diagnostic tests in patients with TB peritonitis were 38\% for an abnormal chest radiograph and 53\% for a positive purified protein derivative test. ${ }^{10}$ Examination of ascites fluid found that lymphocytes predominated in $68 \%$ of cases, and that there was an elevated lactate dehydrogenase level in $77 \%$ of cases and an elevated adenosine deaminase level in 84\%. Mycobacteria were found in 34\% of ascites fluids on culture, but only $3 \%$ of examinations detected organisms by smear. In contrast, the sensitivity of laparoscopic diagnosis was $92 \% .{ }^{10}$ These diagnostic tests for abdominal TB seem unreliable, partly because not every patient develops ascites. Culture of ascites fluid or peritoneal biopsy is the gold standard test. However, even a final diagnosis of pulmonary TB usually takes considerable time; diagnosis of abdominal TB typically takes even longer.

One study from the National Taiwan University Hospital demonstrated that the mean interval between the first day of admission and respiratory isolation for pulmonary TB was 20.5 days. ${ }^{11}$ On the other hand, Bernhard et al. reported that physicians considered abdominal TB in the initial differential diagnosis in only $39 \%$ of cases $(7 / 18)$. Time to specific diagnosis ranged from $<1$ week to $>3$ months. ${ }^{12}$ Chen et al. reported that the average time to diagnosis of abdominal TB in southeastern Taiwan was $48 \pm 10$ days. ${ }^{13}$ Delay in the diagnosis of abdominal TB is therefore more common than for pulmonary TB.

Several studies have observed that the proportion of extrapulmonary TB is higher in individuals who also are infected with HIV ${ }^{14,15}$ and in foreign-born immigrants ${ }^{16}$ in the USA. In addition, a dramatically increasing TB notification rate was observed in sub-Saharan Africa between 1990 and 2005, especially in countries with a high prevalence of adult HIV (>5\%). ${ }^{17}$ The International Standards for Tuberculosis Care states that HIV counselling and testing is indicated for all patients with TB in areas with a high HIV prevalence. ${ }^{18}$ In Taiwan, 15 011 cases of HIV infection had been reported to the 
Taiwan Centers for Disease Control as of 31 December 2007. ' The case burden of HIV infection in Taiwan is significantly lower than that of TB. More data are required to establish the cost-effectiveness of offering HIV testing to TB patients in a region of high TB and relatively low HIV prevalence, such as Taiwan.

We describe this rare case to alert physicians to the fact that with the current trend of increasing HIV prevalence among the Taiwanese, HIV co-infection should be considered when extrapulmonary TB is suspected.

Medical treatment is preferable to treat abdominal TB in patients also infected with HIV, surgery being reserved for complications such as intestinal obstruction, fistula, perforation and haemorrhage. ${ }^{19}$ Our patient was operated on because he had abscesses and peritonitis, which were a complication of TB colitis. With the availability of the surgical specimen, we followed our suspicion and diagnosed HIV, enabling prompt initiation of treatment. Anti-TB medication was started within 10 days, and the patient was discharged in 14 days. The course of diagnosis and treatment was straightforward. After discharge he received medical treatment as an outpatient with a favourable outcome.

\section{Conflict of interest: None.}

REFERENCES

1. Centers for Disease Control, Department of Health, ROC (Taiwan). Statistics of Communicable Diseases and Surveillance Report. 2007. http://www.cdc.gov.tw/lp. asp?ctNode $=2076 \&$ CtUnit $=1144 \&$ EaseDSD $=31 \mathrm{tmp}=5$ (accessed 12 September 2009).
2. Tuberculosis Coalition for Technical Assistance. International Standards for Tuberculosis Care (ISTC). The Hague: Tuberculosis Coalition for Technical Assistance, 2006. http://www.stoptb.org/resource center/assets/documents/istc report. pdf (accessed 12 September 2009).

3. Saltzman DJ, Williams RA, Gelfand DV, Wilson SE. The surgeon and AIDS: twenty years later. Arch Surg 2005; 140: 961-967.

4. Khan $\mathrm{R}$, Abid $\mathrm{S}$, Jafri $\mathrm{W}$, et al. Diagnostic dilemma of abdominal tuberculosis in non-HIV patients: an ongoing challenge for physicians. World J Gastroenterol 2006; 12: 6371-6375.

5. Uygur-Bayramicli O, Dabak G, Dabak R. A clinical dilemma: abdominal tuberculosis. World J Gastroenterol 2003; 9: 1098-1101.

6. Sharma SK, Mohan A. Extrapulmonary tuberculosis. Indian J Med Res 2004; 120: 316-353.

7. Kipp $A M$, Stout $J E_{1}$ Hamilton $C D$, et al. Extrapulmonary tuberculosis, human immunodeficiency virus, and foreign birth in North Carolina, 1993 - 2006. BMC Public Health 2008; 8: 107.

8. Hsu YC, Yang MH, Chen YH, et al. The Epidemiology Characteristics of Extrapulmonary Tuberculosis in Taiwan, 1996-2003. Epidemiol Bull 2007; 23: 231-242.

9. Golden MP, Vikram HR. Extrapulmonary tuberculosis: an overview. Am Fam Physician 2005; 72: 1761-1768.

10. Sanai FM, Bzeizi Kl. Systematic review: tuberculous peritonitis - presenting features, diagnostic strategies and treatment. Aliment Pharmacol Ther 2005; 22: 685-700.

11. Wu YC, Hsu GJ, Chuang KY, et al. Intervals before tuberculosis diagnosis and isolation at a regional hospital in Taiwan. J Formos Med Assoc 2007; 106: 10071012.

12. Bernhard JS, Bhatia G, Knauer CM. Gastrointestinal tuberculosis: an eighteenpatient experience and review. J Clin Gastroenterol 2000; 30: 397-402.

13. Chen HL, Wu MS, Chang WH, et al. Abdominal tuberculosis in southeastern Taiwan: 20 years of experience. J Formos Med Assoc 2009; 108: 195-201.

14. Yang $Z$, Kong $Y$, Wilson $F$, et al. Identification of risk factors for extrapulmonary tuberculosis. Clin Infect Dis 2004; 38: 199-205.

15. Onorato IM, McCray E. Prevalence of human immunodeficiency virus infection among patients attending tuberculosis clinics in the United States. $J$ Infect Dis 1992; 165: 87-92.

16. Talbot $E A$, Moore $M, M c C r a y ~ E$, et al. Tuberculosis among foreign-born persons in the United States, 1993-1998. JAMA 2000; 284: 2894-2900.

17. Reid $A$, Scano $F$, Getahun $H$, et al. Towards universal access to HIV prevention, treatment, care, and support: the role of tuberculosis/HIV collaboration. Lancet Infect Dis 2006; 6: 483-495.

18. Tuberculosis Coalition for Technical Assistance. International Standards for Tuberculosis Care (ISTC). The Hague: Tuberculosis Coalition for Technical Assistance, 2006. http://www.stoptb.org/resource_center/assets/documents/istc_report. pdf (accessed 12 September 2009).

19. Guex AC, Bucher HC, Demartines N, et al. Inflammatory bowel perforation during immune restoration after one year of antiretroviral and antituberculous therapy in an HIV-1-infected patient: report of a case. Dis Colon Rectum 2002; 45: 977978

\title{
GASTRO-INTESTINAL MYCOBACTERIUM $A V I U M$ COMPLEX AS A CAUSE OF ANAEMIA
}

\author{
Annemarie van de Vyver, $B S c, M B C h B, P G D i p T M$ \\ Department of Internal Medicine, University of Pretoria and Kalafong Hospital, Pretoria \\ Adele Visser, $M B$ ChB, DTM\&H, PGDipTM \\ Department of Clinical Pathology, University of Pretoria, and National Health \\ Laboratory Service, Tshwane Academic Division
}

Anaemia is a relatively common finding in HIV-positive patients, with rates (among females) as high as 37\%, compared with their HIV-negative counterparts (17\%). Anaemia of chronic disease plays a very important role in this population group, and is estimated to occur in 18 - 95\% of cases. For this reason, it is imperative to distinguish this condition from other underlying or concurrent causes of anaemia that may warrant treatment. This clinical case illustrates the value of critically evaluating the parameters of a full blood count and haematinic screen, to so determine which patients warrant further workup.

\section{CASE REPORT}

A 43-year-old man, known to be HIV-1 positive, presented to the casualty department at Kalafong complaining of a 2-week history of fatigue, weight loss, night sweats, dysphagia and general malaise. He further described a 3-day history of vomiting and diarrhoea, with no melaena or haematemesis.

At the time of presentation, he had been on firstline highly active antiretroviral (HAART) therapy for 2 years. Despite this the CD4 count on admission was 3 cells/ $\mu \mathrm{l}$. In the light of this finding, non-compliance was suspected. He had previously been diagnosed with 
disseminated Mycobacterium avium-intracellulare by positive blood cultures and had been started on treatment. Owing to side-effects, he had not complied with this treatment regimen either.

On admission he was pyrexial and tachycardic. He was clinically anaemic with no signs of oral hairy leukoplakia or candida. Although abdominal examination was unremarkable (with no hepatomegaly or splenomegaly), he was tender in the epigastric area. Cardiovascular and respiratory examinations were essentially normal.

The full blood count revealed a significant microcytic hypochromic anaemia (haemoglobin $5.8 \mathrm{~g} / \mathrm{dl}$, mean corpuscular volume $68 \mathrm{fl}$ and mean corpuscular haemoglobin $20.4 \mathrm{pg}$ ). The white cell count was normal, but he had thrombocytopenia $\left(30 \times 10^{9} / \mathrm{I}\right)$. Creatinine and electrolyte levels were normal. Liver function tests revealed an isolated mildly raised gamma-glutamyl transpeptidase (GGT) level (70 U/I) and a low albumin level $(16 \mathrm{~g} / \mathrm{l})$. C-reactive protein was elevated at 84.9 $\mathrm{mg} / \mathrm{l}$. Iron studies were also performed and showed low serum iron $(1.3 \mu \mathrm{mol} / \mathrm{l})$ and transferrin $(1.4 \mathrm{~g} / \mathrm{l})$ levels and transferrin saturation (4\%), and a markedly elevated serum ferritin level (1579 $\mu \mathrm{g} / \mathrm{l})$.

As part of the work-up for anaemia, the upper gastrointestinal tract was investigated by endoscopy. This revealed what was clinically judged to be extensive candidiasis throughout the oesophagus. The stomach was normal but the duodenum also had extensively distributed white plaques. A biopsy specimen of these plaques was taken and submitted for histological examination. An HEtE stain was performed (Fig. 1, a). The periodic acid-Schiff (PAS) stain revealed multiple clusters of micro-organisms in the histiocytes (Fig. 1, b). Finally, a Ziehl-Neelsen (ZN) stain was performed, showing large numbers of acid-fast bacilli (Fig. 1, c and $d$ ). A diagnosis of disseminated M. avium complex (MAC) was suggested, as the organisms were found intracellularly. The diagnosis of disseminated MAC was confirmed on a urine sample by molecular techniques.

\section{DISCUSSION}

Patients with advanced HIV-1 disease pose a multitude of challenges in terms of diagnosis and treatment. Anaemia is a relatively common finding in HIV-positive patients, with rates (among females) as high as 37\%, compared with their HIV-negative counterparts (17\%). ${ }^{1}$ The list of possible causes of anaemia in HIV-positive patients is substantial and differentiation is often difficult. Value is certainly added by taking the full blood count results into consideration. A simple distinction between red cell size (reflected in mean corpuscular volume) and red cell haemoglobin content (reflected by mean corpuscular haemoglobin) can significantly contribute to further choices in testing.
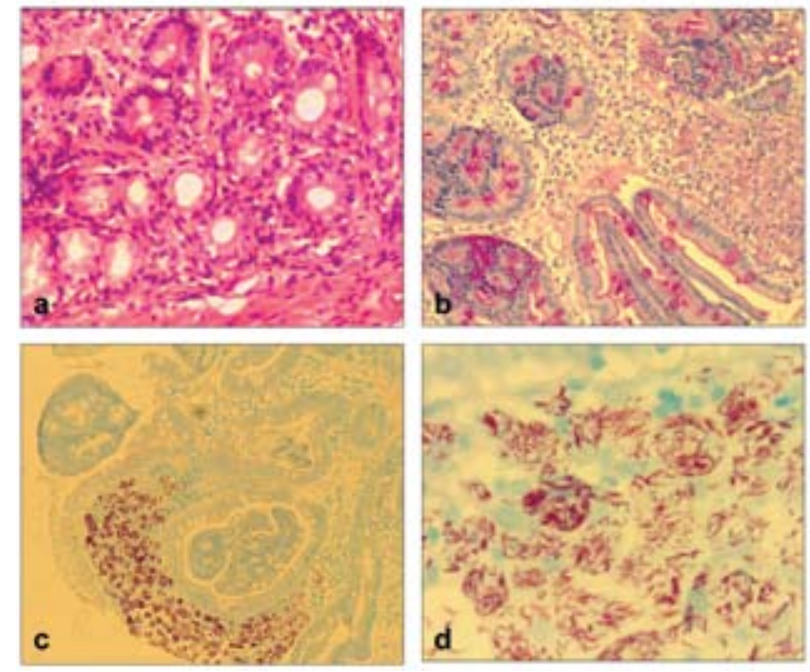

Fig. 1. Sections from white plaques biopsied in duodenum: $a-H \& E$ stain; $b$ - PAS stain showing numerous clumps of organisms in histiocytes; c - ZN stain showing clumps of acid-fast bacilli; $d$ closer view of collection of acid-fast bacilli in ZN stain.

Anaemia of chronic disease plays a very important role in this population group, as inhibition of iron transfer from the reticulo-endothelial cells to the erythroid precursors due to inflammation is estimated to occur in $18-95 \%$ of cases. ${ }^{2}$ For this reason, it is imperative to distinguish this condition from other underlying or concurrent causes of anaemia that may warrant treatment. A haemoglobin level below $8 \mathrm{~g} / \mathrm{dl}$ should prompt further investigation, as anaemia of chronic disease rarely causes World Health Organization grade III or IV anaemia ${ }^{2}$ (grade III $<7.9 \mathrm{~g} / \mathrm{dl}$, grade IV $<6.5 \mathrm{~g} / \mathrm{dl}$ ). ${ }^{1}$ Iron studies may facilitate this process. In both iron deficiency anaemia and anaemia of chronic disease, the serum iron level and transferrin saturation will be reduced. The transferrin level, however, may facilitate in making a distinction as it is typically reduced to normal in anaemia of chronic disease and increased in iron deficiency. A further indicator can be found in serum ferritin levels, which are reduced to below $30 \mathrm{ng} / \mathrm{l}$ (positive predictive value of 92 - 98\%) in iron deficiency, and normal to elevated in anaemia of chronic disease. The inherent confounder with using ferritin is the fact that it acts as an acute-phase reactant and will be elevated beyond its baseline in any inflammatory condition, irrespective of iron status. ${ }^{2}$

The soluble transferrin receptor level may be a useful assay to delineate causes of anaemia. Levels are typically increased in iron deficiency and essentially normal in anaemia of chronic disease, as inflammatory cytokines negatively influence its expression. This can also be very useful if co-existence of both conditions is suspected. However, the assay is not universally offered. The use of various ratios has also been proposed as possibly helpful in determining the underlying cause of anaemia. ${ }^{2}$

The finding and confirmation of iron deficiency should prompt further investigation as to the underlying cause. 
Imaging of the gastro-intestinal tract may be useful, especially if clinical features are suggestive. Of note is the fact that the only feature suggestive of upper gastrointestinal bleeding in our patient was the epigastric tenderness on abdominal examination. It is therefore prudent to have a high index of suspicion. Again, the differential diagnosis in this clinical setting is large and relates to the degree of immunosuppression. ${ }^{3}$

Disseminated MAC is the most common bacterial opportunistic infection among HIV-1-positive patients in the First World. ${ }^{4}$ However, it appears to be less common in our local setting. ${ }^{5}$ It has been postulated that it is caused by the overwhelming presence of $M$. tuberculosis in the South African context. ${ }^{5}$ Patients with a CD4 count below 50 cells/ $\mu$ land possibly high HIV-1 viral loads are at increased risk of MAC infections, which have been shown to be an independent predictor of mortality. For this reason prophylaxis is advocated by some. ${ }^{4}$ It is, however, not included in the current South African National Antiretroviral Treatment Guidelines. ${ }^{6}$

MAC can affect any part of the gastro-intestinal tract, with the duodenum being the most common site. Macroscopic findings are not diagnostic. Biopsy and culture is therefore the mainstay of diagnosis of this condition. $^{3}$

\footnotetext{
REFERENCES

1. Moyle G. Anaemia in persons with HIV infection: Prognostic marker and contributor to morbidity. AIDS Rev 2002; 4: 13-20.

2. Weiss G, Goodnough L. Anemia of chronic disease. N Engl J Med 2005; 352: 1011 1023.

3. Riedel D, Nugent $S$, Gilliam B. Upper gastrointestinal bleeding in a patient with HIV infection. Clin Infect Dis 2009; 48: 368-369.

4. Karakousis $\mathrm{P}$, Moore R, Chaisson R. Mycobacterium avium complex in patients with HIV infection in the era of highly active antiretroviral therapy. Lancet Infect Dis 2004; 4: 557-565.

5. Peter J, Sonderup M. Diagnosing multiple opportunistic infections: The value of liver biopsy. South African Journal of HIV Medicine 2008; Spring: 51-52.

6. Department of Health. National Antiretroviral Treatment Guidelines. Johannesburg: Jacana, 2004.
}

\title{
Invited Comment
}

\section{Abdominal mycobacterial infection in HIV}

The articles in this edition by Kao and Hung and Van de Vyver and Visser both deal with aspects of abdominal mycobacterial infections. Van de Vyver's article highlights the importance of investigating abnormalities that cannot be attributed to HIV infection alone, and demonstrates that abdominal mycobacterial infection may present with a paucity of abdominal symptoms and signs. While tuberculosis (TB) infection is predominant in South Africa, non-tuberculous mycobacteria should always be considered in patients with advanced immunosuppression. The article by Kao demonstrates a more dramatic presentation in a patient with relatively preserved immunity. Notification rates of extrapulmonary TB in South Africa are increasing, and it is likely that more patients will present with abdominal tuberculosis. ${ }^{1}$ Tuberculosis can involve the entire gastro-intestinal tract, from the intra-abdominal organs to the peritoneum. The spectrum of symptoms seen in abdominal TB range from insidious nonspecific complaints that may be mistaken for the constitutional symptoms of HIV infection, to an acute abdomen. ${ }^{2}$ With improved access to antiretrovirals, TB immune reconstitution inflammatory syndrome is being seen more frequently and often involves the abdomen. ${ }^{3}$

In resource-limited facilities, investigations such as abdominal computed tomography scanning and laparoscopic peritoneal biopsy are seldom available. However, abdominal ultrasound, specifically looking for hepatomegaly, ascites, splenic micro-abscesses and intra-abdominal lymphadenopathy, is a useful investigation for assessing HIV-infected patients with suspected abdominal tuberculosis. ${ }^{4}$

Clinicians need to maintain a high index of suspicion for TB in patients with HIV and abdominal symptoms. In the correct clinical setting empiric anti-tuberculosis therapy is warranted. All patients started on anti-tuberculosis therapy need close follow-up until resolution, and those who fail to respond to TB therapy may require further investigation. Nontuberculosis mycobacterial infection should be considered in patients with advanced immune deficiency.

While abdominal tuberculosis in HIV-infected patients is best managed with standard TB therapy and anti-retrovirals, complications such as obstruction, perforation and large abscess formation may require surgical intervention. ${ }^{2}$ Depending on clinical presentation, early consultation with the surgeons is essential and if required surgery should not be delayed.

\author{
A D Black \\ Department of Medicine, Chris Hani Baragwanath Hospital and University of the Witwatersrand, Johannesburg \\ REFERENCES \\ 1. WHO Global Report on Tuberculosis 2009. http://apps.who.int/globalatlas/predefinedReports/TB/PDF_Files/zaf.pdf (accessed 24 June 2010 ). \\ 2. Lazarus AA, Thilagar B. Abdominal tuberculosis. Dis Mon 2007; 53: 32-38. \\ 3. Meintjes G, Rabie J, Wilkinson RJ, Cotton MF. Tuberculosis-associated immune reconstitution inflammatory syndrome and unmasking of tuberculosis by antiretroviral \\ therapy. Clin Chest Med 2009; 30(4): 797-810. \\ 4. Sinkala E, Gray S, Zulu I, et al. Clinical and ultrasonographic features of abdominal tuberculosis in HIV positive adults in Zambia. BMC Infect Dis 2009; 9: 44.
}

